\title{
Correlation Analysis of Sprint Performance and Reaction Time Based on Double Logarithm Model
}

\author{
Jing Zhang, ${ }^{1}$ Xin-Yu Lin, ${ }^{2}$ and Su Zhang $\mathbb{D}^{3}$ \\ ${ }^{1}$ Faculty of Physical Education, Shanghai International Studies University, Shanghai 200083, China \\ ${ }^{2}$ School of Applied Mathematics, Anhui University of Finance and Economics, Bengbu 233030, China \\ ${ }^{3}$ Department of Physical Education, Anhui University of Finance and Economics, Bengbu 233030, China
}

Correspondence should be addressed to Su Zhang; zs0625@163.com

Received 23 December 2020; Revised 13 January 2021; Accepted 28 January 2021; Published 9 February 2021

Academic Editor: M. Irfan Uddin

Copyright ( $\odot 2021$ Jing Zhang et al. This is an open access article distributed under the Creative Commons Attribution License, which permits unrestricted use, distribution, and reproduction in any medium, provided the original work is properly cited.

In sprint track events, the starting reaction time is an important professional capacity of the athletes, and it is closely related to their performance. This study examines the reaction time and the results of the male and female sprinters participating in the World Athletics Championships from 2011 to 2019 in the $100 \mathrm{~m}, 200 \mathrm{~m}, 100 \mathrm{~m}$, and $110 \mathrm{~m}$ hurdles. The researchers used least squares estimation, multivariate analysis of variance, and other methods and theories to construct a double logarithmic model and a multivariate analysis of a variance model. The researchers used Econometrics Views and SPSS software programs to analyze the correlation between the performance and the starting reaction time, as well as the patterns in the changes of the reaction time of athletes of both genders in different types of and rounds in the competitions. Research results show that there is a direct correlation between the reaction time and the performance, and the degrees of correlation vary depending on the gender of the athlete, year of competition, type of competition, and round of competition. There is a correlation between the foul types and the type of competition, but there is no correlation between foul types and the gender of the athlete. The research results are science-based and are of practical value and thus can be used as a reference by coaches in sprint running to offer more professional guidance to the athletes.

\section{Introduction}

Track and field is one of the most-watched athletic events, which embodies the spirit of mankind to challenge the limit and continue to make breakthroughs. And the Olympic 100meter race is the particular focus of the global audiences [1]. With the continuous improvement of sprint performance, sprint performance indicators are getting closer and closer to the human limit. For high-level athletes, among whom the physical and technical gaps are shrinking, the difference in performance is sometimes narrowed down to only 0.001 second.

The reaction time of sprint runners is one of the factors that affect their performance. The research of Helmick and Tellez shows that reaction time contributes about $1 \%$ to $2 \%$ to the overall performance of the sprinters, and in a 100 meter race, about $5 \%[2,3]$. The reaction time is not only affected by innate factors, such as the athlete's nerve and muscle types, but is also influenced by training and psychological factors [4]. In sprinting events, the reaction time is influenced by the following factors: the time required for the start signal stimulus to reach the receptors, the delay time for the receptors to convert the stimulus into nerve signals, neurotransmission and processing, the activation of the delayed muscles, the soft tissue adaptability, and the external measurement parameters [5].

The reaction time refers to the shortest time from the reception of the stimulus to the response. It has a significant influence on the action execution and performance of the athletes in different sport events $[6,7]$. Studies have shown that there is a latency of the reaction time, which can be shorten via professional training, and thus the results can be improved. So, reaction training is a breakthrough point for world-class athletes in pursuit of new world record [7]. 
Studies have shown that all top athletes have very short reaction time, and the reaction time is significantly positively correlated to the final results. As the distance increases, the reaction time increases significantly with a noticeable difference between male and female athletes [8-10]. In the research on the correlation between the reaction time and the performance, some scholars believe that there is a positive correlation between these two factors for top sprinters. From the 100 -meter race to the 200 -meter race, the average reaction time can increase linearly by $10 \mathrm{~ms}$. And this positive correlation can be observed in both male and female $100 \mathrm{~m}$ races [11-13]. However, some studies have shown that there is a low or even no correlation between the reaction time and the performance $[14,15]$. In the research on the gender difference in the reaction time, the researcher looked at the 100-meter race of the 16th Asian Games and found a gender difference in the reaction-performance correlation [16]. However, some other researchers studied the reaction time and the results of male and female athletes running the 100-meter, 200-meter, and 400-meter events in the 2012 London Olympic Games, and they did not find any gender difference. Other researchers have also examined the correlation between reaction time and performance of athletes of both genders who participated in the 100-meter race, 100-meter hurdle running, and 110-meter hurdle running in Sydney, Athens, and Beijing Olympic Games. They found that the correlation is gender-based only for the 100 -meter race. The same study also shows a variation in the correlation among the three Olympics. The reaction timeperformance correlation in the Beijing Olympic Games is found to be much stronger than those of the Sydney and Athens Olympics. In a word, previous research results are not consistent, and some are contradictory [17-19].

The starting reaction time is a unique method of measuring the starting reaction speed, that is, the time from the moment the signal is sent (by the automatic timing starting gun) to the time when the athlete's pedaling force on the starting device reaches $300 \mathrm{~Pa}$. The monitoring system presets a corresponding pressure threshold according to the specific events, and the time from the starting signal to the time when the pedaling pressure reaches the threshold is the official reaction time for the athletes. Given this, the Competition and Technical Rules formulated by the World Athletics stipulate that "starting foul monitors must be used in sprint competitions above Category $\mathrm{C}$ to reduce misjudgments or missed judgments caused by manual penalties, and among them, $400 \mathrm{~Pa}$ for adult men and 300-350 $\mathrm{Pa}$ for adult women, starting reaction time $<0.1 \mathrm{~s}$, will be regarded as a foul [20]."

From a holistic and systematic perspective, this study examines the complete data of five consecutive World Athletics Championships sprint competitions from 2011 to 2019 to analyze factors that influence the reaction time of sprint athletes, such as gender, the type of the competition, and the round of competition, etc. The researchers analyzed the correlation between the reaction time and the performance and found out the dynamic changing patterns. The research findings can be used to confirm existing conclusions or question some commonly held views, and can point out the research direction and research path for future research studies.

\section{Correlation Analysis: Reaction Time and Competition Performance}

2.1. Research Design and Problem Analysis. First of all, the researchers processed and analyzed the sample data and then selected the reaction time as the independent variable and the competition performance as the dependent variable. Next, in order to study the influence of the reaction time on the performance, the researchers established a double logarithmic model. Finally, the researchers tested the heteroscedasticity of the model and used the weighted least squares method to modify the model.

\subsection{Double Logarithmic Model of Athletes' Competition Performance and Reaction Time}

2.2.1. Use of Symbols. The main variables involved in this article are as follows: (1) $Y$ the total running time; (2) $X_{1}$ the reaction time; (3) $\varepsilon$ the fitting error; (4) $\beta_{1}$ the flexibility of the total running time concerning the reaction time; (5) $D_{i}$ gender dummy variable.

\subsubsection{Model Establishment}

Sample Selection and Data Processing. First, the researchers selected the reaction time and the running time as independent and dependent variables, respectively. And then, we filtered the data by eliminating three types of special values in the total running time, including disqualified results, withdrawal during the game, and DNS waiver.

Model Setting. In addition to selecting the variables in the model, the regression model should reflect the basic economic relationship between the variables. To reflect the degree of influence of the reaction time on the performance, the authors established a double logarithmic model. The model setting form is

$$
\operatorname{Ln} Y=\alpha_{0}+\beta_{1} \operatorname{Ln} X_{1}+\varepsilon .
$$

Among them, $Y$ is the total running time, $X_{1}$ is the reaction time, $\varepsilon$ is the fitting error, and the slope $\beta_{1}$ measures the elasticity of the explained variable $Y$ concerning the explanatory variable $X_{1}$, or the average percentage in the change of the performance when the reaction time changes by $1 \%$. By observing the magnitude of the slope coefficient, it is possible to observe the contribution rate of the reaction time to the performance.

Model Test. Because the sample data are cross-sectional, it is likely that there is heteroscedasticity. Then, the White test is performed. With the assistance of Econometrics Views, through the residual square estimated by least squares, an auxiliary regression is formed for the constant, explanatory variable, explanatory variable square, and its cross product. 
And the $P \leq 0.0001$, so the null hypothesis is rejected, indicating that the model has heteroscedasticity.

Modification of the Model. The weighted least squares method is used to correct the heteroscedasticity, and the final model estimation result is

$$
\begin{aligned}
\operatorname{Ln} Y= & 3.5109+0.4644 \operatorname{Ln} X_{1} \\
& \cdot(0.0790)(0.0465), \\
t= & (44.4301)(11.1484), \\
R^{2}= & 0.9787, \\
\overline{R^{2}}= & 0.9689 \\
F= & 124.2873 \\
D W= & 2.1619 .
\end{aligned}
$$

At the $5 \%$ significance level, the model fitting effect is good, and the estimated equation can pass the significance test.

2.3. Result Analysis. After leaving the starting block, it takes a world-class 100 -meter sprinter $5 \%$ of the total race time to reach $1 / 3$ of his or her maximum speed. The starting performance of the sprinter is closely related to the total race time [5]. Through the analysis of the fitted model, the elastic coefficient of the athlete's reaction time to the athletic performance is 0.4644 . That is to say, when the athlete's reaction time increases by one unit, the average running time increases by 0.4644 seconds. Therefore, in short-distance track and field events, there is a correlation between the reaction time and the competition result, but it is not absolute. The results of this research are consistent with the conclusions of Michel, Plilanids, Zhai Huanan et al., and others on the correlation between the reaction time of track and field athletes and their performance $[15,21,22]$.

\section{Correlation Analysis: Gender and Reaction Time}

3.1. Research Design. First, the authors studied the influence of the athlete's gender on the reaction time, and we introduce dummy variables in an additive manner to reflect gender differences. Then, we performed the ordinary least squares estimation through Econometrics Views, and the results can reflect the degree of influence of gender on the reaction time.

3.2. Research Method: Least Squares Method. Based on the research on the relationship between athletes' competition performance and reaction time, to study the influence of gender on athletes' starting reaction, an additive method is used to introduce dummy variables to reflect gender differences. Select the starting reaction time as the explained variable, and set the form of the model as

$$
Y_{1}=\alpha_{0}+\beta_{0} D_{i}+\varepsilon
$$

among them, $D_{i}= \begin{cases}1, & \text { male athletes, } \\ 0, & \text { female athletes }\end{cases}$

3.3. Result Analysis. The researchers used Econometrics Views for ordinary least squares estimation, and the model estimation results obtained are as follows:

$$
\begin{aligned}
Y_{1}= & 0.1707-0.0067 D_{i} \\
& \cdot(0.0009)(0.0012), \\
t= & (188.8617)(-5.3005), \\
R^{2}= & 0.9118, \\
\overline{R^{2}}= & 0.9114, \\
F= & 98.0901, \\
P \leq & 0.0001 .
\end{aligned}
$$

At the $5 \%$ significance level, the model fitting effect is good, and the estimated equation can pass the significance test. According to the model, the average starting reaction time of men is 0.0067 second faster than that of women. Thus, there is a direct correlation between the reaction time and the gender of the athlete. Although the current false start threshold set in the starting block system adopted by the IAAF does not differentiate male and female athletes, some scholars suggest that we set different thresholds for male and female athletes in short-distance races, considering the known differences in strength and strength development rates between men and women [23, 24].

\section{Correlation Analysis: Reaction Time and Year of the World Championship}

4.1. Research Design. Based on the research on the correlation between the athlete's gender and the reaction time and the results of the five World Championships from 2011 to 2019 , two-factor analysis of variance was performed on the reaction time. Then, the researchers standardized the data to make it eligible, used SPSS for statistical analysis, and then analyzed the correlation between the year of competition and the reaction time.

4.2. Research Methods. To find out the features of the reaction time of male and female athletes in different competition years, the researchers used the reaction time as the dependent variable, the year and gender as independent variables, and conducted variance analysis. The results are shown in Table 1.

4.3. Result Analysis. In the reaction time, the year of competition and the gender of the athlete have an influence on the results. And the main effect of year and gender reaches a significant level $(P \leq 0.001)$. It shows that in addition to gender, the year of competition is also correlated with the reaction time of the athletes. According to the average value and standard deviation of the reaction time of the athletes each year (see Table 2), the reaction time of both 
TABLE 1: Analysis results of the reaction time and year of the World Championship.

\begin{tabular}{lccccc}
\hline Source & Type III sum of squares & Degree of freedom & Mean square & $F$ & Significance \\
\hline Revised model & $208.117^{\mathrm{a}}$ & 9 & 23.124 & 25.258 & 0.000 \\
Gender & 19.525 & 1 & 19.525 & 21.327 & 0.000 \\
Years & 165.694 & 4 & 41.424 & 45.246 & 0.000 \\
Gender $*$ year & 6.807 & 4 & 1.702 & 1.859 & 0.115 \\
Total & 2366.000 & 2367 & - & - & - \\
Revised total & 2366.000 & 2366 & - & - \\
\hline
\end{tabular}

TABLE 2: The average value and standard deviation of the athlete's reaction time each year.

\begin{tabular}{lccc}
\hline Gender & Year & Average value & Standard deviation \\
\hline \multirow{4}{*}{ Male } & 2011 & 0.193 & 0.043 \\
& 2013 & 0.179 & 0.044 \\
& 2015 & 0.166 & 0.043 \\
& 2017 & 0.163 & 0.034 \\
Female & 2019 & 0.161 & 0.030 \\
& 2011 & 0.196 & 0.051 \\
& 2013 & 0.188 & 0.040 \\
& 2015 & 0.174 & 0.036 \\
& 2017 & 0.169 & 0.036 \\
\end{tabular}

male and female athletes are shortening by different degrees year by year. And the reaction time in 2011 is significantly longer than those in the following four competitions. Besides, there are significant differences among the reaction time in different World Championships. This shows that with the training getting more professional and sciencebased, the reaction capacity of athletes has increased significantly accordingly.

\section{Correlation Analysis: Race Type and Reaction Time}

5.1. Research Design. Based on the research on the correlation between the athlete's gender, year of competition, and the reaction time, the researchers conducted a threefactor variance analysis on the reaction time in different types of race, including straight, corner, hurdle, and allaround competitions. Then, the researchers used SPSS to analyze the influence of these factors on the reaction time and analyzed the correlation between the race type and the reaction time.

5.2. Research Methods. This research is to identify the timeorder characteristics of the reaction time and running time in different race types. The previous study has proved that there is a significant influence of gender and year of competition on the reaction time. So, with this research, the researchers used the reaction time as the dependent variable, and the year, gender, and race type as independent variables for the variance analysis. The results are shown in Table 3. In the starting reaction time, the main effects of gender and race type as well as the interaction between the year and the race type reached a significant level $(P \leq 0.001)$.
5.3. Result Analysis. Studies have suggested that the reaction time accounts for about $1-2 \%$ of the overall running time of sprinters (Baumann, 1980; Helmick, 2003). The time to sprint against 200-meter athletes is longer than that of 100meter or 110-meter hurdle athletes, and the impact of starting reaction on the performance of 200-meter athletes is lower than that of 100-meter or 110-meter hurdle athletes [25].

The researchers used SPSS for statistical analysis and processing and found that when it comes to the reaction time, the main effect of the year and the race type and the interaction between the year and race type both reached a significant level $(P \leq 0.001)$. Further multiple comparison results show that for male athletes, the reaction time in the 100 -meter race is significantly shorter than those in the 200meter race and the 110-meter hurdle, and for female athletes, the reaction time in the 100-meter hurdle is significantly shorter than those in the 100-meter race and the 200-meter race, and the reaction time in 200-meter race is significantly shorter than that in the 100-meter race. So, it can be concluded that the reaction time vary significantly in different types of race.

\section{Correlation Analysis: Round of Competition and Reaction Time}

6.1. Problem Analysis. Based on the research studies on the correlation between the athlete's gender, competition year, race type, and the reaction time, the researchers conducted a multifactor analysis of variance on the influence of round of competition (preliminaries, semifinals, and finals) on the reaction time. The researchers used SPSS for statistical analysis to identify the degree of difference in the reaction time of all athletes in different rounds of the competition. In 
TABle 3: Analysis of differences in reaction time of athletes in different race types.

\begin{tabular}{lccccc}
\hline Source & Type III sum of squares & Degree of freedom & Mean square & $F$ & Significance \\
\hline Revised model & $359.066^{\mathrm{a}}$ & 28 & 12.824 & 14.849 \\
Intercept & 0.061 & 1 & 0.061 & 0.071 & 0.000 \\
Gender & 18.738 & 1 & 18.738 & 21.698 \\
Years & 142.395 & 5 & 28.479 & 32.978 & 0.000 \\
Project class & 50.569 & 2 & 25.285 & 29.279 & 0.000 \\
Gender $*$ year & 6.756 & 4 & 1.689 & 1.956 \\
Gender $*$ project class & 6.840 & 2 & 3.420 & 3.960 & 0.090 \\
Year $*$ project class & 44.988 & 8 & 5.623 & 0.019 \\
Gender $*$ year $*$ item & 24.381 & 6 & 4.063 & 4.712 & 0.000 \\
\hline
\end{tabular}

other words, the researchers studied the correlation between the round of competition and the reaction time.

Then, based on the research studies on the correlation between the athlete's gender, competition year, race type, and reaction time, a multifactor analysis of variance was carried out on finalists to examine their reaction time in the preliminaries, semifinals, and the finals. The researchers studied the degree of difference in the reaction time of final round competitors in different rounds of the competition.

\subsection{Problem Solving}

6.2.1. Correlation Analysis: Round of Competition and Reaction Time. The researchers tried to identify the correlation between the reaction time and the different rounds of competition. With the previous research studies, it can be concluded that there is a significant influence of gender, year of competition, and race type on the reaction time. So, the researchers used the reaction time as a dependent variance and used the year of competition, gender of athlete, race type, and the round of competition as independent variances for variance analysis. The results are shown in Table 4.

Research on the correlation between round of competition and the reaction time shows that when it comes to the reaction time, the main effects of year of competition, race type, round of competition, and gender, as well as the interaction among year of competition, gender, race type, and round of competition, have all reached a significant level. Further multiple comparison results show that for male athletes, the reaction time in the preliminary rounds is significantly higher than those in the semifinal and final competition, and the reaction time in the semifinal is significantly higher than that in the final competition. For female athletes, the reaction time in the semifinal is significantly higher than those in the preliminaries and the final competition, and the reaction time in the preliminary rounds is significantly higher than that in the final competition. It can be inferred that there is a significant correlation between the round of competition and the reaction time for all athletes.

6.2.2. Correlation Analysis: Reaction Time of Finalists in Different Rounds of Competition. Based on the research on the correlation between the round of competition and the reaction time, the researchers further narrowed the scope down to finalists. The researchers conducted a normality test of their reaction time. As shown in Figures 1 and 2, there are large fluctuations in the residual, and many of the absolute differences exceed 0.05 , making the data nonnormal. The researchers then conducted a logarithm transformation, and the $P-P$ diagram is shown in Figures 3 and 4 . At this time, the fluctuation is small, and the absolute difference does not exceed 0.05 . The transformed data meet the conditions for multifactor analysis of variance.

In the previous research, it was found that there is a significant correlation between the round of competition and the reaction time, just as there are significant correlations between gender, race type, and the reaction time. To identify the features of reaction time of finalists in different rounds of competition, the researchers used the reaction time as the dependent variance and used the year, gender, race type, and round of competition as independent variance for variance analysis. The results are shown in Table 5.

The research on the correlation between different competition rounds and reaction time of the finalists shows that the main effects of the year and race type and the interaction of the year and race type reached a significant level $(P \leq 0.001)$. While gender and round of competition types have not reached a significant level.

It can be seen that the reaction time of sprinters in the preliminaries is significantly higher than that of the semifinals and finals, and the reaction time is correlated with the final competition results. The athletes' running time is relatively longer in the preliminaries and is significantly shorter in the semifinals and finals. The reaction time of the finalists is significantly shorter than other athletes, showing a stronger competiveness and better on-the-spot performance of the top athletes. It is also worth mentioning that for the finalists, their reaction time in the final round is longer than their reaction time in the preliminaries and the semifinal. This is probably related to the zero-tolerance false start rule adopted by the IAAF. With this rule, the athletes are more cautious in the final round of competition, and the fear of being disqualified due to a false start will consume more psychological energy and therefore will make a direct impact on the reaction time $[21,26]$.

\section{Correlation Analysis: Reaction Time in Different Foul Types}

7.1. Problem Analysis. In addition to the difference in abilities between sprinters, which affects the starting reaction 
TABLE 4: Analysis of the difference in reaction time of athletes in different rounds of competition.

\begin{tabular}{|c|c|c|c|c|c|}
\hline Source & Type III sum of squares & Degree of freedom & Mean square & $F$ & Significance \\
\hline Revised model & $500.509^{\mathrm{a}}$ & 93 & 5.382 & 6.470 & 0.000 \\
\hline Intercept & 2.671 & 1 & 2.671 & 3.212 & 0.073 \\
\hline Years & 75.738 & 4 & 18.935 & 22.764 & 0.000 \\
\hline Project class & 32.597 & 3 & 10.866 & 13.063 & 0.000 \\
\hline Gender & 19.253 & 1 & 19.253 & 23.147 & 0.000 \\
\hline Competition type & 47.544 & 3 & 15.848 & 19.054 & 0.000 \\
\hline Year* project class & 32.374 & 12 & 2.698 & 3.243 & 0.000 \\
\hline Year $*$ gender & 1.525 & 4 & 0.381 & 0.458 & 0.006 \\
\hline Year $*$ competition type & 9.320 & 11 & 0.847 & 1.019 & 0.007 \\
\hline Project class $*$ gender & 0.949 & 1 & 0.949 & 1.141 & 0.006 \\
\hline Project class $*$ competition type & 8.063 & 6 & 1.344 & 1.616 & 0.009 \\
\hline Gender * stage & 0.517 & 3 & 0.172 & 0.207 & 0.002 \\
\hline Year $*$ project class $*$ gender & 3.851 & 4 & 0.963 & 1.158 & 0.008 \\
\hline Year $*$ project class $*$ competition type & 27.169 & 24 & 1.132 & 1.361 & 0.003 \\
\hline Year $*$ gender $*$ competition type & 14.020 & 8 & 1.752 & 2.107 & 0.002 \\
\hline Project class $*$ gender $*$ competition type & 8.794 & 2 & 4.397 & 5.286 & 0.005 \\
\hline Year $*$ project class $*$ gender $*$ competition type & 4.598 & 7 & 0.657 & 0.790 & 0.006 \\
\hline
\end{tabular}

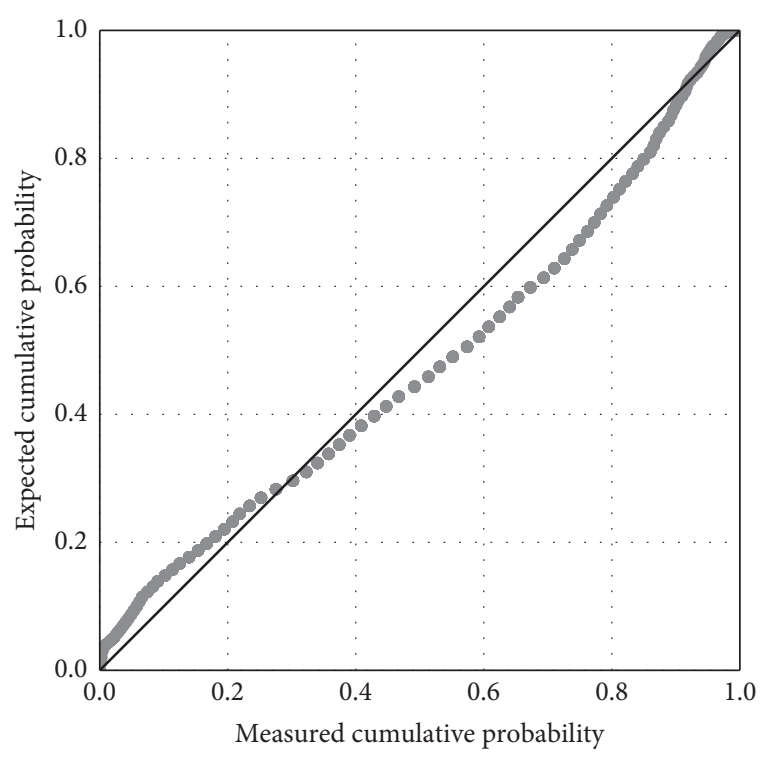

Figure 1: Normal $P-P$ diagram during reaction time.

time, other factors, such as changes to the disqualification rules, will also affect the starting reaction time of the athletes [24]. To identify the correlation between foul type and reaction time, we can analyze the factors which have closely relationship to foul type. Firstly, we can filter the data to count the types of fouls that occurred in each competition, and then we use the contingency table analysis to explore the correlation between gender, year, project class, competition type, and foul type (DQ|DNF|DNS). Then, combine these factors to carry out descriptive statistical analysis of the foul type. Finally, we can carry out correlation analysis of foul type and starting the reaction.

\subsection{Problem Solving}

7.2.1. Foul Statistics of Each Competition. We look up the data of the World Athletics Championships and find out the fouls in the five games from 2011 to 2019, and the data are shown in Table 6.

From the data in Table 6, it can be seen that the record of no results due to abstention (DNS) is 0 , and the records of canceled match results (DQ) and halfway retired (DNF) have been recorded only about 4-9 times during 2011-2015. The number of fouls in the race between 2015 and 2017 is 0 . In the sprint race, the pace is faster and the general failure to finish (DNF) may be due to injury or other physical factors. The reason for disqualification (DQ) is generally because of false started. World Athletics revised the new starting rules to prevent athletes from deliberately starting to affect the state of other athletes. The decline in fouls also shows that the game is becoming more and more fair and reasonable.

7.2.2. Correlation Analysis: Type of Foul and Gender. In this part, the type of foul and gender chi-square test in 2011, 2013 , and 2015 all exceeded $20 \%$ of the theoretical frequency $<5$, so Fisher's exact test was selected. The test results are shown in Tables 7 and 8 . There were no significant correlations in gender for the two types of fouls of 2011, 2013, and 2015: cancellation of competition results and withdrawal during the competition.

7.2.3. Correlation Analysis: Type of Foul and Race Type. In this part, the type of athletes fouls in 2011, 2013, and 2015 and the chi-square test of the competition event exceeded $20 \%$ of the theoretical frequency $<5$, so Fisher's exact test was chosen. The test results are shown in Tables 9 and 10. There is no significant difference in the race type, where the two types of fouls occurred during the cancellation of the competition results and withdrawal during the competition in 2013 and 2015, and the types of fouls that occurred during the race. There are significant differences in the race type that occurred between the two fouls in the 2011 session. 


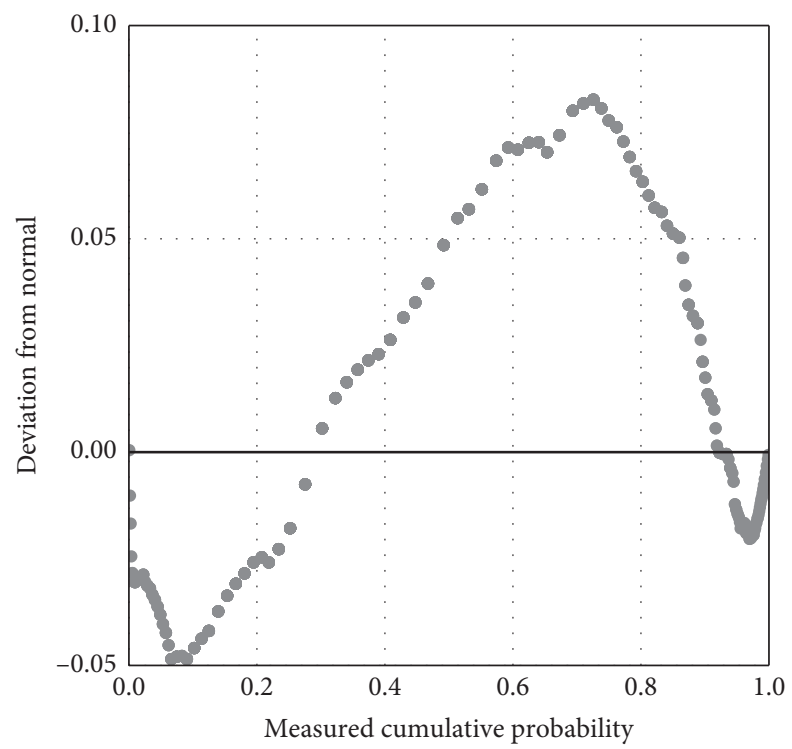

Figure 2: Detrended normal $P-P$ diagram during reaction time.

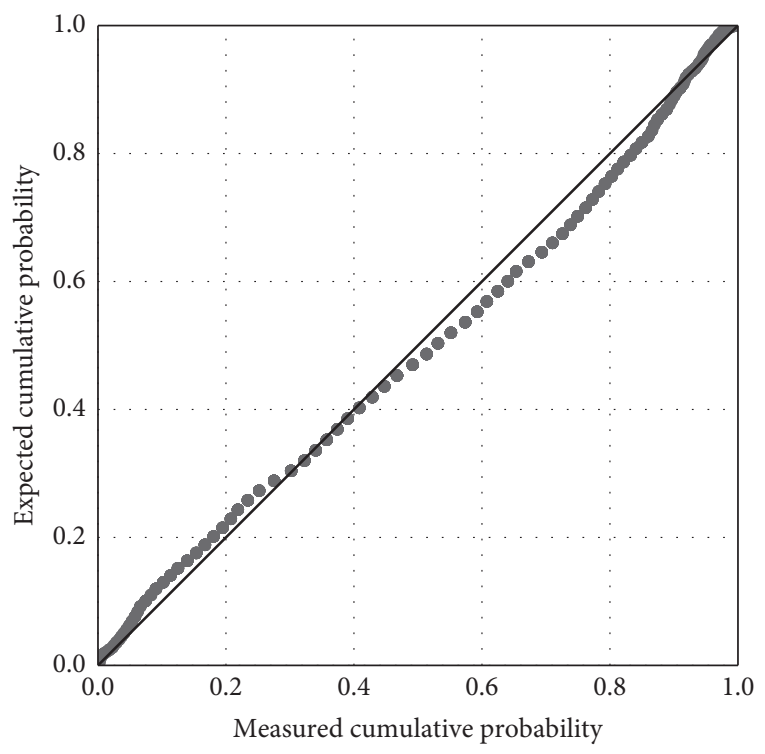

Figure 3: The normal $P-P$ diagram of the Reaction time after natural logarithm transformation.

7.2.4. Correlation Analysis: Type of Foul and Round of Competition. Multiple independent sample contingency table chi-square test result selections: all theoretical frequencies $\geq 5$, see Pearson chi-square results; more than $20 \%$ of theoretical frequencies $<5$ or at least one theoretical frequency $<1$, see Fisher's exact test results. In this part of 2011, 2013, and 2015, the chi-square test in the foul and round of competition exceeded $20 \%$ of the theoretical frequency $<5$, so Fisher's exact test was chosen. In the data used in this article, in 2013, when RESULT is DQ or DNF, there is no Preliminary in Competition; in 2015, when RESULT is DQ or DNF, there is no Preliminary and Final in Competition. After testing, the results shown in Tables 11 and 12

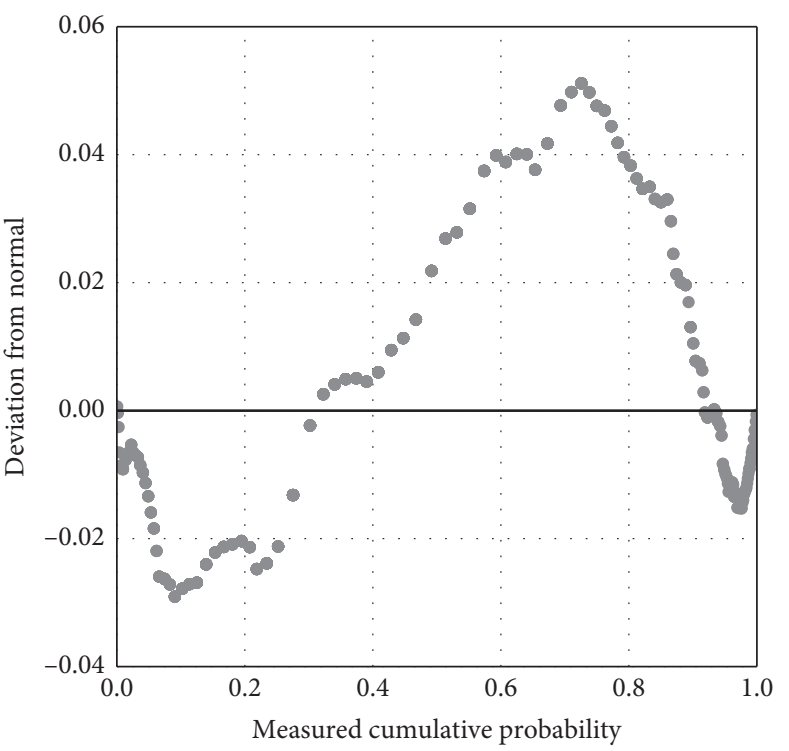

FIgURE 4: Detrended normal $P-P$ diagram Reaction time after natural logarithm transformation.

show that there is no significant difference in the competition types where the two fouls occur during the cancellation of the 2011 and 2013 games and the withdrawal during the game. There are significant differences in the round of competition where the two fouls occur in the 2015 session.

7.2.5. Result Analysis. In 2011, 2013, and 2015, there were two types of fouls: cancellation of game results and withdrawal during the game. There were no fouls in 2017 and 2019. In the analysis of the foul type and the type of the competition, the type of foul and the type of the game, and the contingency table of the type of foul and gender, the characteristics of the foul are as follows. In the first round 
TABLE 5: Differences in the reaction time of the finalists in different rounds of competition.

\begin{tabular}{|c|c|c|c|c|c|}
\hline Source & Type III sum of squares & Degree of freedom & Mean square & $F$ & Significance \\
\hline Revised model & $6.008^{\mathrm{a}}$ & 88 & 0.068 & 3.812 & 0.000 \\
\hline Intercept & 2875.342 & 1 & 2875.342 & 160570.362 & 0.000 \\
\hline Competition type & 0.094 & 2 & 0.047 & 2.614 & 0.074 \\
\hline Project class & 0.923 & 3 & 0.308 & 17.180 & 0.000 \\
\hline Years & 2.000 & 4 & 0.500 & 27.928 & 0.000 \\
\hline Gender & 0.061 & 1 & 0.061 & 3.430 & 0.064 \\
\hline Competition type $*$ project class & 0.150 & 6 & 0.025 & 1.392 & 0.215 \\
\hline Competition type $*$ year & 0.205 & 8 & 0.026 & 1.428 & 0.181 \\
\hline Competition type $*$ gender & 0.087 & 2 & 0.043 & 2.425 & 0.089 \\
\hline Project class $*$ year & 0.707 & 12 & 0.059 & 3.292 & 0.000 \\
\hline Project class $*$ gender & 0.048 & 1 & 0.048 & 2.706 & 0.100 \\
\hline Year $*$ gender & 0.076 & 4 & 0.019 & 1.054 & 0.378 \\
\hline Competition type $*$ project class $*$ year & 0.777 & 24 & 0.032 & 1.808 & 0.010 \\
\hline Competition type $*$ project class $*$ gender & 0.100 & 2 & 0.050 & 2.783 & 0.062 \\
\hline Competition type $*$ year $*$ gender & 0.235 & 8 & 0.029 & 1.639 & 0.110 \\
\hline Project class $*$ year $*$ gender & 0.073 & 4 & 0.018 & 1.019 & 0.397 \\
\hline Competition type $*$ project class $*$ year $*$ gender & 0.091 & 7 & 0.013 & 0.729 & 0.047 \\
\hline
\end{tabular}

TABle 6: Fouls in each competition from 2011 to 2019.

\begin{tabular}{lccccc}
\hline Time/Year & 2011 & 2013 & 2015 & 2017 & 0 \\
\hline DNF & 5 & 4 & 5 & 0 & 0 \\
DQ & 6 & 4 & 9 & 0 & 0 \\
DNS & 0 & 0 & 0 & 0 \\
\hline
\end{tabular}

TABLE 7: Contingency table of foul type and gender.

\begin{tabular}{|c|c|c|c|c|c|}
\hline \multirow{2}{*}{ Year } & \multirow{2}{*}{\multicolumn{2}{|c|}{ Result and types of fouls }} & \multicolumn{2}{|c|}{ Gender } & \multirow{2}{*}{ Total } \\
\hline & & & Men & Women & \\
\hline \multirow{3}{*}{2011} & \multirow{3}{*}{ RESULT } & DQ & 5 & 1 & 6 \\
\hline & & DNF & 3 & 2 & 5 \\
\hline & & & 8 & 3 & 11 \\
\hline \multirow{3}{*}{2013} & \multirow{3}{*}{ RESULT } & DQ & 2 & 2 & 4 \\
\hline & & DNF & 3 & 1 & 4 \\
\hline & & & 3 & 3 & 8 \\
\hline \multirow{3}{*}{2015} & \multirow{3}{*}{ RESULT } & DQ & 8 & 1 & 9 \\
\hline & & DNF & 2 & 3 & 5 \\
\hline & & & 10 & 4 & 14 \\
\hline
\end{tabular}

TABLE 8: Chi-square analysis of the foul type and gender.

\begin{tabular}{lcccc}
\hline Fisher's exact test & Value & Degree of freedom & Progressive significance (bilateral) & Exact significance (two-sided) \\
\hline 1 & 0.130 & 1 & 0.505 & 0.545 \\
2 & 1.002 & 1 & 0.897 & 1.000 \\
3 & 1.079 & 1 & 0.441 & 0.095 \\
\hline
\end{tabular}

and the semifinals, the probability of fouling is higher, and the probability of fouling in the final is less, which decreases year by year; fouls are more likely to occur in the 200-meter and 110-meter hurdles. There are significant differences in the types of fouls in the two types of fouls in the 2011 session. Cancellation of race results is more likely to occur in the 100 meters, and halfway retirement is more likely to occur in the 200 meters. There is no significant correlation in gender between the two fouls (DQ and DN) in 2011, 2013, and 2015. 
TABLE 9: Contingency table of foul type and race type.

\begin{tabular}{|c|c|c|c|c|c|c|c|}
\hline \multirow{2}{*}{ Year } & \multirow{2}{*}{\multicolumn{2}{|c|}{ Results and types of fouls }} & \multicolumn{4}{|c|}{ Project class } & \multirow{2}{*}{ Total } \\
\hline & & & $100 \mathrm{~m}$ & $100 \mathrm{H}$ & $110 \mathrm{H}$ & $200 \mathrm{~m}$ & \\
\hline \multirow{3}{*}{2011} & & DQ & 5 & 0 & 1 & 0 & 6 \\
\hline & RESULT & DNF & 0 & 1 & 1 & 3 & 5 \\
\hline & & & 5 & 1 & 2 & 3 & 11 \\
\hline \multirow{3}{*}{2013} & & DQ & 2 & 1 & 0 & 1 & 4 \\
\hline & RESULT & DNF & 1 & 0 & 2 & 1 & 4 \\
\hline & & & 3 & 1 & 2 & 2 & 8 \\
\hline \multirow{3}{*}{2015} & & $\mathrm{DQ}$ & 9 & 1 & 4 & 4 & 9 \\
\hline & RESULT & DNF & 5 & 3 & 2 & 0 & 5 \\
\hline & \multicolumn{2}{|c|}{ Total } & 14 & 4 & 6 & 4 & 14 \\
\hline
\end{tabular}

TABLE 10: Chi-square analysis of foul type and race type.

\begin{tabular}{lcccc}
\hline Fisher's exact test & Value & Degree of freedom & Progressive significance (bilateral) & Exact significance (two-sided) \\
\hline 1 & 8.552 & 3 & 0.620 & 0.009 \\
2 & 3.153 & 3 & 0.824 & 0.657 \\
3 & 4.357 & 3 & 0.694 & 0.157 \\
\hline
\end{tabular}

TABLE 11: Contingency table of foul type and round of competition.

\begin{tabular}{|c|c|c|c|c|c|c|c|}
\hline \multirow{2}{*}{ year } & \multirow{2}{*}{\multicolumn{2}{|c|}{ Result and types of fouls }} & \multicolumn{4}{|c|}{ Competition type } & \multirow{2}{*}{ Total } \\
\hline & & & Final & Preliminary & Round 1 & Semifinal & \\
\hline \multirow{3}{*}{2011} & \multirow{2}{*}{ RESULT } & DQ & 2 & 2 & 1 & 1 & 6 \\
\hline & & DNF & 3 & 0 & 0 & 2 & 5 \\
\hline & \multicolumn{2}{|c|}{ Total } & 5 & 2 & 1 & 3 & 11 \\
\hline \multirow{3}{*}{2013} & \multirow{3}{*}{ RESULT } & DQ & 0 & - & 2 & 2 & 4 \\
\hline & & DNF & 1 & - & 1 & 2 & 4 \\
\hline & & & 1 & - & 3 & 4 & 8 \\
\hline \multirow{3}{*}{2015} & \multirow{3}{*}{ RESULT } & DQ & - & - & 8 & 1 & 9 \\
\hline & & DNF & - & - & 1 & 4 & 5 \\
\hline & & & - & - & 9 & 5 & 14 \\
\hline
\end{tabular}

TABLE 12: Types of fouls and chi-square tests in the competition phase.

\begin{tabular}{lcccc}
\hline Fisher's exact test & Value & Degree of freedom & Progressive significance (bilateral) & Exact significance (two-sided) \\
\hline 1 & 3.136 & 3 & 0.800 & 0.610 \\
2 & 1.408 & 2 & 0.937 & 1.000 \\
3 & 32 & 1 & 0.391 & 0.023 \\
\hline
\end{tabular}

\section{Conclusion}

A sprinter's competition starts before the first visible movement or generation of strength in the lower limb muscles, so the reaction ability of the sprinter is very important $[27,28]$. With the shortening of the race distance, the influence of the reaction time on the overall performance increases [29]. At the same time, with the science-based improvement of the training, the coaches and athletes are paying more attention to the training and improvement of starting reaction in short-distance straight track events. This has enhanced the importance of starting reaction in the whole competition process.
In the study of the correlation between the sprinters' performance and the reaction time, the researchers used theories and methods including the least square estimation and multivariate analysis of variance and constructed the double logarithmic model and a multivariate analysis of a variance model, and the researchers then used Econometrics Views and SPSS software programs to analyze the data. With these methods, the authors can make further breakthroughs on the basis of the previous studies, which are homogenous and have their limitations. This study yielded better fitting results through nonmass data, can be promoted, has a higher accuracy, and thus is practical and realistic. 
The research results show that there is a correlation between the reaction time and competition results, but the correlation is not absolute. There is a gender difference in the reaction time, and the reaction time of male athletes is shorter than that of the female athletes. There is a significant difference among the reaction time in different years, with the reaction time decreasing year by year. There is a significant difference in the reaction time of athletes in different types of race. The reaction time in the preliminaries is significantly longer than that in the semifinals and the finals. The reaction time of finalists is significantly shorter than that of the other athletes. However, for the finalists, their reaction time in the final round is longer than their reaction time in the preliminaries and the semifinal. The foul type is correlated to the type of competition, but not to gender.

The study examined the reaction time, its changing patterns, gender difference, and foul types in different types of race; it also looked at the correlation between the reaction time and the competition results. By conducing overall, systemic analysis of the correlation between the reaction time and the competition results, the researchers identified the dynamic changing patterns and explained the degree of influence of the reaction time on the sprinters' competition results. The results are science-based and are of practical value. While providing more research data on the basis of the previous inconsistent studies, this study also serves as a more professional, scientific reference for training for coaches of sprinting events.

\section{Data Availability}

The data in this article came from World Athletics performance statistics for sprint events at the World Athletics Championships from 2011 to 2019.

\section{Conflicts of Interest}

The authors declare that they have no conflicts of interest.

\section{Acknowledgments}

This research was supported by Humanities and Social Sciences Research Major Project of the Education Department of Anhui Province (SK2017A0452, SK2019A1161), Anhui Philosophy Social Sciences Planning Project (AHSKY2017D11), and Provincial Foundation for Excellent Young Talents of Colleges and Universities of Anhui Province (gxgwfx2018030).

\section{References}

[1] N. E. Bezodis, S. Willwacher, and A. I. T. Salo, "The biomechanics of the track and field sprint start: a narrative review," Sports Medicine, vol. 49, no. 9, pp. 1345-1364, 2019.

[2] K. Helmick, "Biomechanical analysis of sprint start positioning," Track Coach, vol. 163, pp. 5209-5214, 2003.

[3] T. Tellez and D. Doolottle, "Technique analysis: sprinting from start to finish," Track Technique, vol. 88, pp. 2802-2805, 1984.
[4] D. P. Zhu and A. L. Lin, "Research on the relationship between the starting reaction time and sports performance of excellent indoor short-distance track and field athletes," Journal of Chengdu Sport University, vol. 45, no. 5, pp. 80-86, 2019.

[5] M. Ishikawa, P. V. Komi, and J. A. Salmi, "IAAF sprint start research project: is the $100 \mathrm{~ms}$ limit still valid?" New Studies in Athletics, vol. 24, no. 1, pp. 37-47, 2009.

[6] E. Zemkova, "Differential contribution of reaction time and move-ment velocity to the agility performance reflects sport specific de-mands," Human Movement, vol. 17, no. 2, pp. 94-101, 2016.

[7] G. Hong, X. U. Fu, and X. U. Shi, "Breakthrough points of recreating world record of men's 100 meter run," Journal of Wuhan Institute of Physical Education, vol. 35, no. 1, pp. 101-102, 2001.

[8] H. B. Jang, "Research on relation between reaction time and performance of sprint and hurdle," Journal of Capital University of Physical Education and Sports, vol. 28, no. 5, pp. 469-475, 2016.

[9] A. Delalija and V. Babić, "Reaction time and sprint results in athletics," International Journal of Performance Analysis in Sport, vol. 8, no. 2, pp. 67-75, 2008.

[10] J. N. Luo, H. C. Xie, and D. U. S. Wu, "Research on the features of pre-start back kick time-pressure curve of sprint-based on the sample of the 100-metre male sprinters in the 16th asian games," Journal of Capital University of Physical Education and Sports, vol. 35, no. 5, pp. 60-63, 2012.

[11] M. Stevenson, "The sprint start: save as many split-seconds as you can on the start and you'll be in pretty good shape at the finish," Coach and Athletic Director, vol. 66, no. 8, pp. 18-20, 1997.

[12] V. Babic and A. Delalija, "Reaction time trends in the women's sprint and hurdle events at the 2004 olympic games," New Studies in Athletic, vol. 24, no. 1, pp. 49-57, 2009.

[13] G. Paradisis, E. Zacharogiannis, and A. Smirniotoy, "Correlation of reaction time and performance in $100 \mathrm{~m}$ sprint running," Medicine and Science in Sports and Exercise, vol. 38, no. 5 , p. 518, 2006.

[14] P. Moravec, J. Ruzicka, and P. Susanka, "The 1987 International athletic foundation/IAAF scientific project report: time analysis of the 100 metres events at the II world championships in athletics," New Studies in Athletics, vol. 3, no. 3, pp. 61-96, 1988.

[15] S. Michel and J. Jarcer, "The start is (almost) everything in sprint performance," Track Coach, vol. 160, p. 5121, 2002.

[16] J. N. Luo and H. C. Xie, "Research on the different start reaction time of the male and female athletes in the $100 \mathrm{~m}$ dash of the 16th asian games," China Sport Science and Technology, vol. 47, no. 4, pp. 14-18, 2011.

[17] R. Pavlovi, K. Idrizovi, and M. Vrci, "Differences in time of start reaction and achieved result in the sprint disciplines in the finals of the olympic games in London," Sports Science and Health, vol. 4, no. 1, pp. 5-19, 2014.

[18] P. Theophilos, A. Kasabalis, and N. Mantzouranis, "Start reaction time and performance at the sprint event in the olympic games," Kinesiology, vol. 44, no. 1, pp. 67-72, 2012.

[19] H. N. Zhai and C. C. Feng, "Characteristics of time order of start reaction time and sports performance of elite athletes in $60 \mathrm{~m}$ running and $60 \mathrm{~m}$ hurdles," Journal of Wuhan Institute of Physical Education, vol. 52, no. 11, pp. 95-100, 2018.

[20] WA-World Athletics, Competition and Technical Rules 2020, Monaco, WA, USA, 2020, https://www.worldathletics.org/ about-iaaf/documents/technical-information. 
[21] Th. Plilanids, N. Manzulanis, and A. Kasabalis, "Start reaction time and performance at the sprint events in world athletics championships," International Journal of Performance Analysis in Sport, vol. 12, no. 1, pp. 112-118, 2012.

[22] H. N. Zhai and C. C. Feng, "The time sequence characteristics of start reaction and athletic performance of elite athletes in $60 \mathrm{~m}$ and $60 \mathrm{~m}$ hurdles," Journal of Wuhan Institute of Physical Education, vol. 52, no. 11, pp. 96-101, 2018.

[23] D. B. Lipps, A. T. Galecki, and J. A. Ashton-Miller, "On the implications of a sex difference in the reaction times of sprinters at the beijing olympics," PLoS One, vol. 6, no. 10, 2011.

[24] K. C. Brosnan, K. Hayes, and A. J. Harrison, "Effects of falsestart dis-qualification rules on response-times of elite-standard sprinters," Journal of Sports Sciences, vol. 35, no. 10, pp. 1-7, 2016.

[25] N. E. Bezodis, A. IT. Salo, and G. Trewartha, "Relationships between lower-limb kinematics and block phase performance in a cross section of sprinters," European Journal of Sport Science, vol. 15, no. 2, pp. 1-7, 2014.

[26] C. Englert and A. Bertrams, "The effect of ego depletion on sprint start reaction time," Journal of Sport and Exercise Psychology, vol. 36, no. 5, pp. 506-515, 2014.

[27] A. Mero and P. V. Komi, "Reaction time and electromyographic activity during a sprint start," European Journal of Applied Physiology and Occupational Physiology, vol. 61, no. 12, pp. 73-80, 1990.

[28] A. Boisnoir, L. Decker, B. Reine, and F. Natta, "Validation of an integrated experimental set-up for kinetic and kinematic three-dimensional analyses in a training environment," Sports Biomechanics, vol. 6, no. 2, pp. 215-223, 2007.

[29] D. P. Zhu and A. L. Li, "The study on the relationship between elite athletes start reaction time and sport performance in indoor short distance running," Journal of Chengdu Sport University, vol. 45, no. 5, pp. 80-86, 2019. 